\title{
Improving the diagnosis and management of urinary tract infections at Surrey and Sussex NHS Trust
}

\author{
Author: Shuaib Quraishi
}

\section{Aims}

Urinary tract infection (UTI) is one of the most commonly diagnosed infections among hospitalised patients. We know from previous audit data that we are inappropriately diagnosing and inappropriately prescribing antibiotics for UTIs, which can lead to side effects and harm to patients. Audit data suggest that $30 \%$ of patients treated for a UTI actually meet national guideline criteria. Urine samples were sent in $69 \%$ uncatheterised episodes where the clinical criteria for UTI were not fulfilled. There is an overreliance on urine dips for diagnosing UTI in elderly patients, leading to inappropriate antibiotic prescriptions. The aim is to reduce the number of inappropriate antibiotic prescription use, create awareness and improve patient outcomes.

\section{Methods}

Important stakeholders were identified which included microbiologists, the chief of medicine, infection prevention, control and antibiotic stewardship (IPCAS) group, the lead pharmacist, infection control nurses, consultants, geriatricians and junior doctors. A semi-qualitative questionnaire using SurveyMonkey was used to explore doctors' views on their knowledge in the diagnosis and management of UTIs.

A further audit concentrated on diagnosis of UTI in elderly patients who presented through the emergency department within a 2-week period. The data collected was compared against national clinical guidelines.

\section{Results}

There were 700 patients over the age of 75 years that presented to the emergency department. Thirty-nine had a diagnosis of UTI recorded in their clinical notes. Only $20 \%$ of these met the national guideline criteria for diagnosing a UTI; $10 \%$ of patients had a 'positive urinalysis' as the only rationale recorded for their diagnosis.

The doctor questionnaire had 52 responses and we found that although doctors had good background knowledge on diagnostic criteria for managing UTIs it was not put into practice. It also identified there was a clear need for guidance, awareness and

Author: Royal College of Physicians chief registrar, Surrey and Sussex NHS Trust training. Fifty per cent of people did not use any form of training to assist them in diagnosing and managing UTIs.

\section{Conclusion}

There is a clear need for training. We plan to incorporate this into junior doctor induction as well as at various educational meetings. Posters have been designed and will be placed in key areas. Our geriatricians have worked with a multiprofessional faculty to develop a range of podcasts, including ones on UTIs and urinary incontinence in older people, and these are freely available educational resources for the wider multidisciplinary team. A longer-term vision is to develop a module that is composed of podcasts, YouTube videos and articles that form a blended learning approach.

\section{Conflict of interest statement}

Nil. 